\section{The Structural and Chemical Analyser - A New Analytical Technique for SEM}

\author{
A.D.Brooker*, T.Prusnick**, R.M.Jarvis***, \\ R.Goodacre $^{* * *}$, R.Bennett*, C.J.Dawe* ${ }^{*}$ D.J.Leak ${ }^{*}$, \\ M.R.Hill*, M.J.Lainchbury* \\ ${ }^{*}$ Renishaw plc, Gloucestershire, UK \\ **Renishaw Inc., Illinois, USA \\ *** Department of Chemistry, UMIST, Manchester, UK \\ Alan.Brooker@renishaw.com
}

\section{Introduction}

Renishaw's structural and chemical analyser (SCA) provides new analytical techniques for scanning electron microscopes (SEM). For many years energy dispersive X-ray analysis (EDS) has been the default in-SEM analytical technique, and whilst it can provide fast and accurate data from any samples, it can only provide elemental information about the sample being analysed. For materials applications, assumptions about stoichiometry may enable accurate characterisation, but in most instances "chemistry by guesswork" does not provide an acceptable solution. Furthermore, EDS is rather insensitive to light elements - atomic number 11 (sodium) and below, which severely limits its applicability to organic chemistry and life sciences.

Renishaw has developed the structural and chemical analyser (SCA) for SEM, which is a novel accessory combining the imaging advantages of SEM with the analytical capabilities of optical spectroscopy. The principal analytical method offered by the SCA is Raman spectroscopy, but the system also supports photoluminescence $(\mathrm{PL})$ and cathodoluminescence $(\mathrm{CL})$ spectroscopies.

Until now, Raman spectroscopy has used a conventional optical microscope to project a small laser spot onto the surface of a sample, and to collect efficiently the Raman signal. The SCA uses in-SEM retractable collection optics to introduce the laser light and focus it into a micrometre-order sized spot on the sample, and to collect the Raman signal. The collection optics are introduced between the objective lens and the sample, and a small aperture in them allows the electron beam to reach the sample so that secondary electron images can be viewed whilst Raman spectroscopy is being carried out. Retractable optics are used for two reasons, principally so that the performance of the SEM is not compromised - when the optics are fully-retracted there are no restrictions to the SEM's use or specification. Secondly, the optics can be rapidly moved to a "standby" position so that techniques requiring line-of-sight to the sample (e.g. backscattered electron imaging - BEI, and energydispersive X-ray analysis - EDS) can be used. The SCA has been designed to allow the collection optics to be repositioned in $\mathrm{X}, \mathrm{Y}$, and $\mathrm{Z}$ with sub-micrometre accuracy so that the Raman analytical position is precisely maintained. The laser light and Raman signal are both transmitted to the SCA using fibre optics so that a range of spectrometer models can be used, and these can be situated a distance from the SEM.

The SCA allows the advantages of SEM and optical spectroscopy to be combined in a single instrument. SEM provides excellent resolution, depth of field, and a range of contrast mechanisms that make the identification of regions of interest fast and accurate. Optical spectroscopies allow the chemical, structural, and electrical nature of samples to be explored, as summarised in table 1.

\section{Experimental}

The potential of the SCA is illustrated by the examination of a number of samples from different disciplines.

Table 1 - Table showing the information that can be recorded using the SCA

\begin{tabular}{|c|c|c|c|}
\hline Spectroscopy & Spectral feature & Information content & Examples \\
\hline Raman & $\begin{array}{l}\text { Characteristic peak positions } \\
\text { (frequencies) }\end{array}$ & $\begin{array}{l}\text { Chemical composition and identification } \\
\text { of materials, allotropes, and polymorphs }\end{array}$ & $\begin{array}{l}\mathrm{Fe}_{2} \mathrm{O}_{3} \text { (haematite), } \mathrm{Fe}_{3} \mathrm{O}_{4} \text { (magnetite), } \\
\text { crocidolite, chrysotile, rutile, anatase }\end{array}$ \\
\hline Raman/CL/PL & $\begin{array}{l}\text { Peak shifts (changes in } \\
\text { characteristic frequency) }\end{array}$ & Stress and strain in the matrix & $\begin{array}{l}\text { Si shows } a=10 \mathrm{~cm}^{-1} \text { Raman shift per } \\
1 \% \text { strain }\end{array}$ \\
\hline $\mathrm{Raman} / \mathrm{CL} / \mathrm{PL}$ & Peak width & Crystal quality & $\begin{array}{l}\text { Plastic deformation, inclusions, } \\
\text { residual stress }\end{array}$ \\
\hline Raman/CL/PL & Intensity & Amount of material & $\begin{array}{l}\text { Coating thickness - SiGe, diamond- } \\
\text { like carbon (DLC) }\end{array}$ \\
\hline $\mathrm{CL} / \mathrm{PL}$ & $\begin{array}{l}\text { Characteristic peak positions } \\
\text { (wavelengths) }\end{array}$ & $\begin{array}{l}\text { Band gap voltage, presence of carriers, } \\
\text { impurities and vacancies }\end{array}$ & GaN, GaAs, CVD diamond \\
\hline
\end{tabular}

Figure 1-A secondary electron image of diamond grown around a tungsten core, Raman spectra collected at the positions shown demonstrate that the crystallinity of the diamond layer improves as further layers are deposited

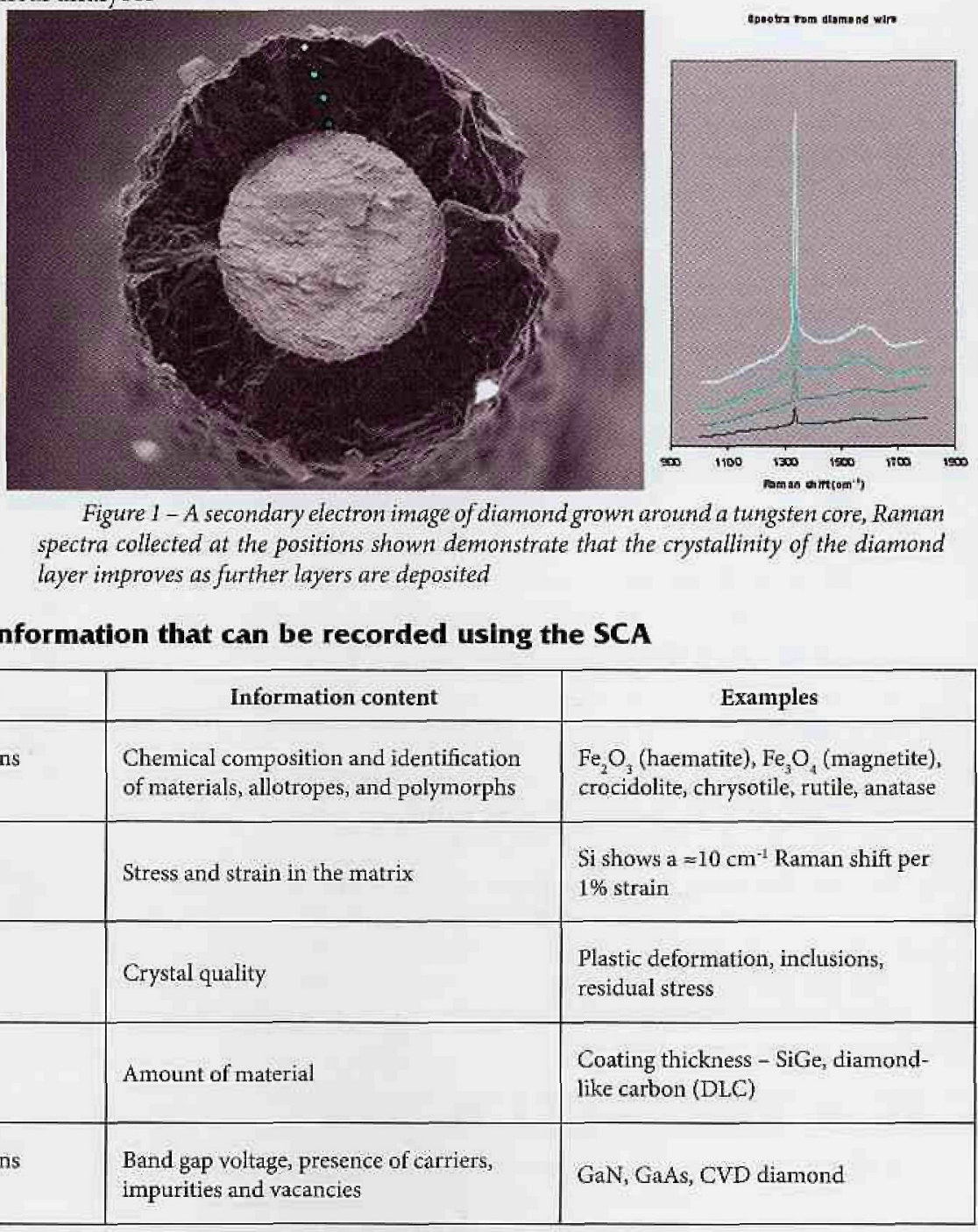




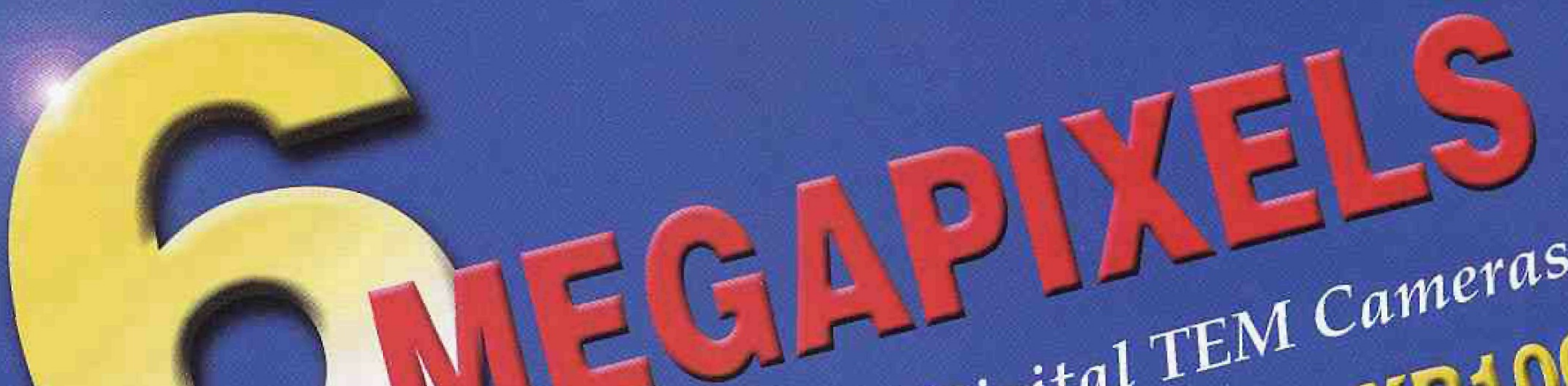

IETEMARMTON

TEMTEMATION

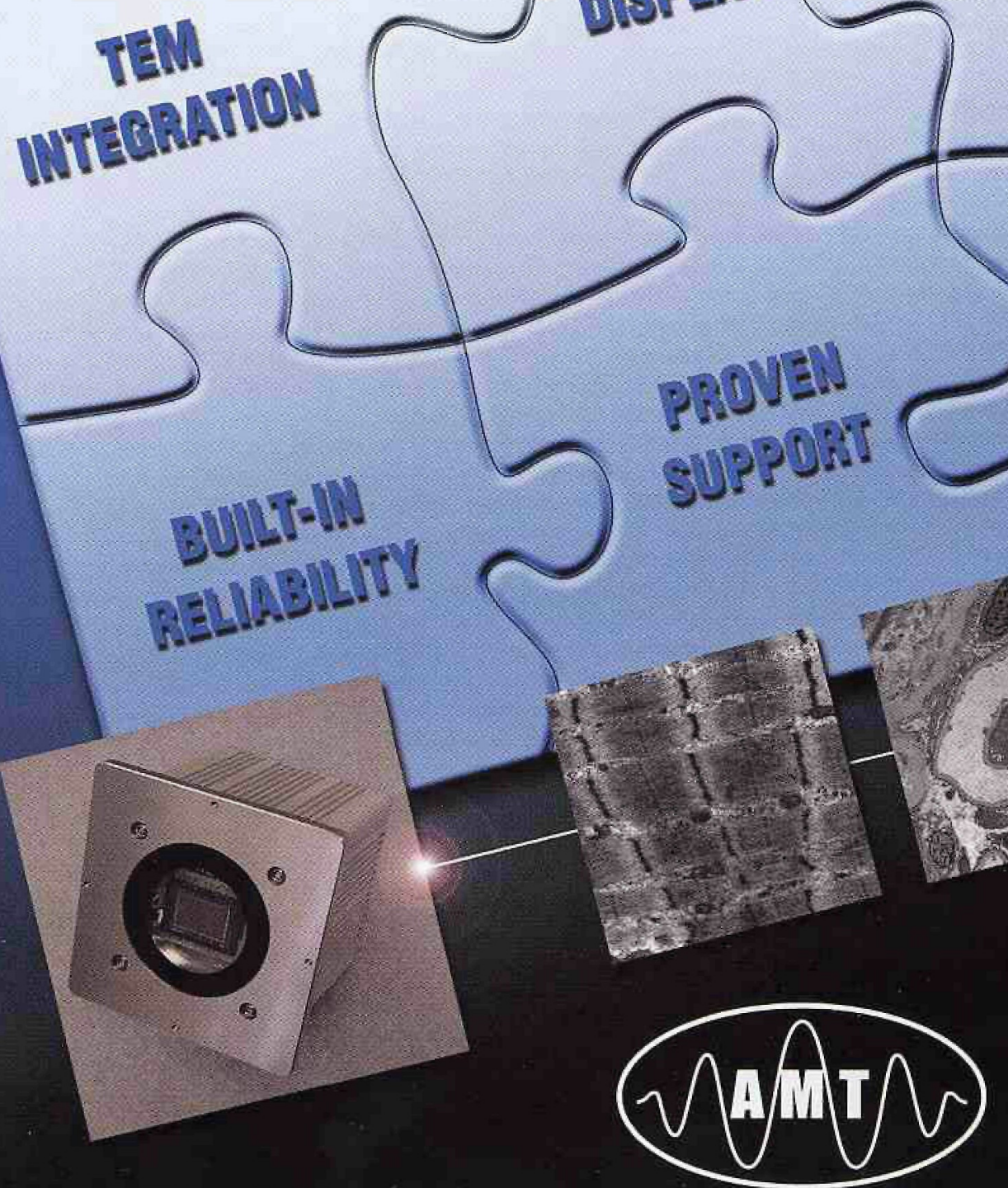

LARQEST FIELD of VIIEW

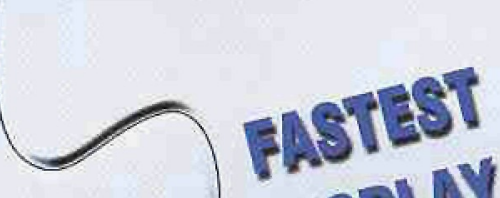

AMT's

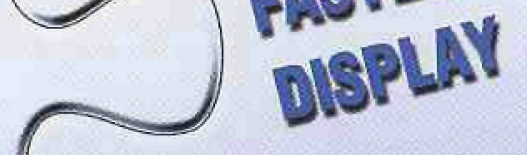
DISPLAN

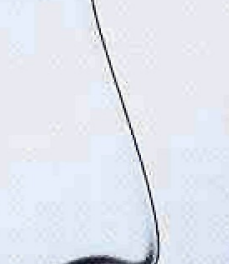


CL spectrum of GaN

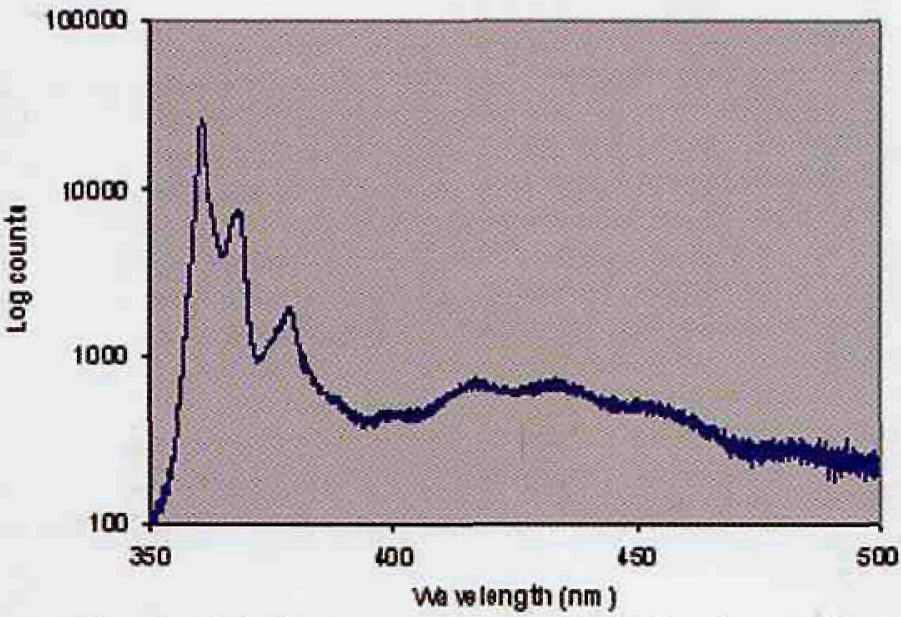

Figure 2 - Cathodoluminescence spectrum of $\mathrm{GaN}$ collected at $-170^{\circ} \mathrm{C}$

Figure 1 shows an SEM image of diamond grown around a tungsten wire core. Raman spectra were taken close to the wire core, and at various positions towards the last deposited layer (outermost), as shown in the figure. The data show that diamond was being deposited (from the characteristic Raman peak - amorphous carbon, graphite, and carbon nanotubes have significantly different spectra). The spectra also show that the quality of the layer improves as subsequent layers are deposited (the diamond peak becomes narrower, sharper, and has improving signal to background indicating that the degree of crystallinity is increasing, and the amount of inclusions is decreasing).

Figure 2 shows a cathodoluminescence (CL) spectrum from gallium nitride (GaN) collected at $-170^{\circ} \mathrm{C}$. These data are difficult to collect using conventional optical microscopy because inefficient long working distance optical lenses are needed to accommodate cooling stages, and expensive laser and custom optics are required to work in the UV regime. With CL the electron beam can excite the band gap for most materials, and the vacuum environment means that low temperature observations can be made without the sample "icing up".

The SCA was used for the examination of bacteria labelled using metallic nanoparticles (a silver colloid). An e-coli culture was mixed with a colloidal solution, which associates itself with proteins in the bacterial coating - the labelled culture was then simply dried down onto an SEM stub. Take-up of the label, however, is fairly inefficient and optical microscopy is unable to discriminate between labelled and unlabelled regions making analysis a rather random operation. Atomic number contrast from backscattered electron imaging in the SEM (see figure 3) easily distinguishes between areas with and without the metallic nanoparticles. EDS analysis of the sample reveals only aluminium from the stub and a weak carbon signal (from the bacteria). Raman spectra from the sample using the SCA (see figure 4) show that where the label was present a strong Raman spectrum could be obtained - these spectra also show the surface enhanced Raman spectroscopy (SERS) effect, which both quenches fluorescence, and greatly enhances the Raman signal levels. Areas without silver labels showed only fluorescence. Unlike many SERS experiments, using the SEM to identify labelled regions gave highly repeatable data, which, when combined with cluster analysis allowed discrimination between the e-coli spectra, and those from another similar bacterial strain.
The SCA has also been successful in characterising both aqueous and thermal metallic oxide films, and the identification and distribution of active ingredients, excipients and fillers in pharmaceutical samples.
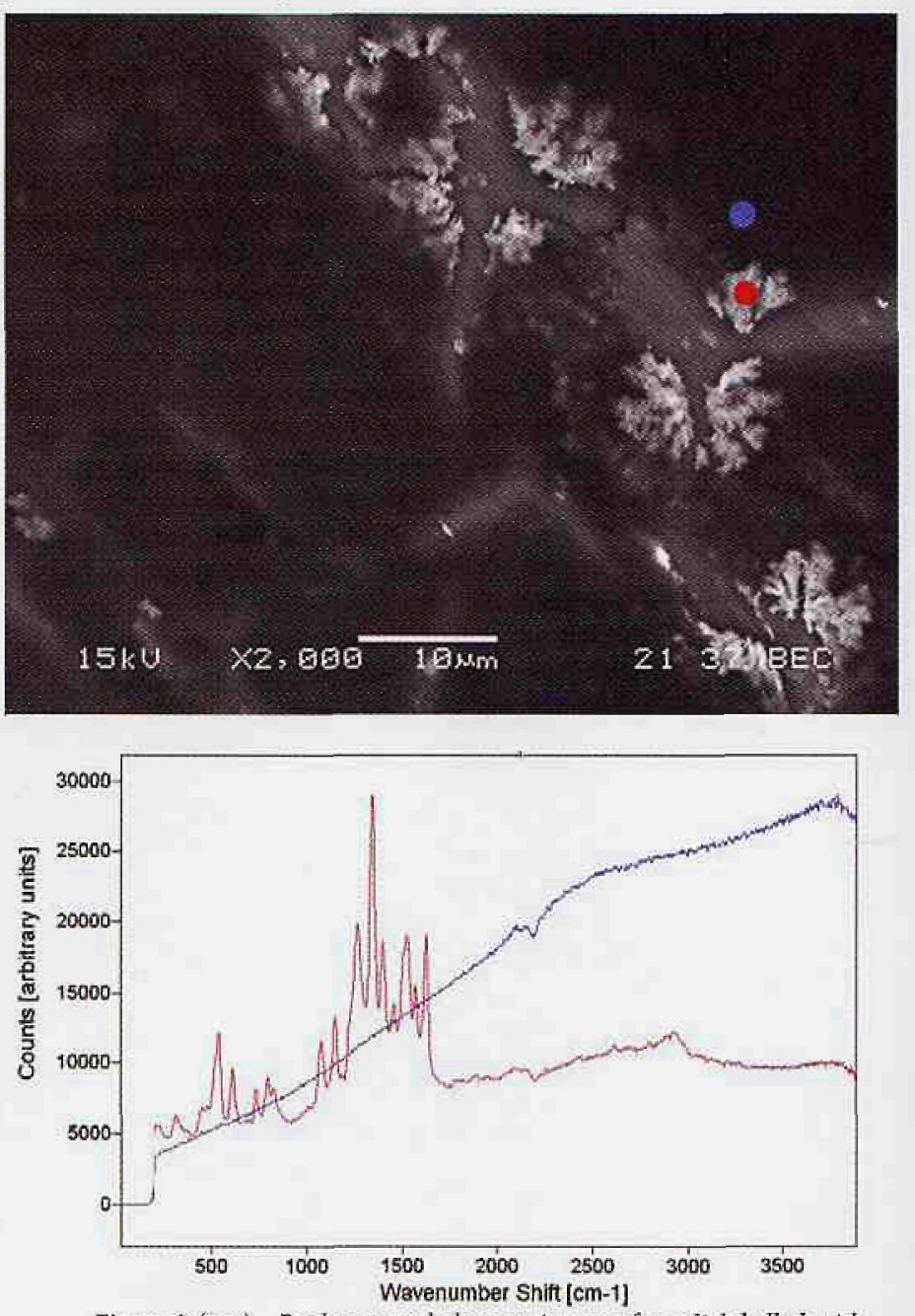

Figure 3 (top) - Backscattered electron image of e-coli labelled with metallic nanoparticles

Figure 4 (bottom) - Raman spectra from labelled (red) and unlabelled (blue) e-coli.

\section{Conclusion}

The combination of scanning electron microscopy and optical spectroscopy realised by the SCA provides scientists with a new tool, which revolutionises analytical work. A single instrument can now be used to characterise unambiguously objects of interest, overcoming the difficulties associated with relocating them, and ensuring that they have not been altered when transferring them between multiple instruments.

\section{Publications:}

"SEM-Raman Spectroscopy," Inst.Phys.Conf.Ser.No 180, Paper presented at Microscopy of Semiconducting Materials, p573, 2003

"Renishaw's structural and chemical analyser (SCA) for scanning electron microscopes," Renishaw product note SPD/PN/096

"A study of single-wall carbon nanotubes using Renishaw's structural and chemical analyser for scanning electron microscopes" Renishaw application note SPD/AN/092

"Characterisation of particulates on semiconductors using the structural and chemical analyser for SEM" Renishaw application note SPD/AN/093

Renishaw's publications are available on www.renishaw.com/spectroscopy 


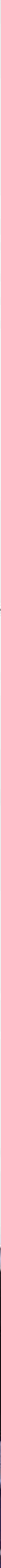

